\title{
IDENTIFICACIÓN Y VALORACIÓN NEUROPSICOLÓGICA DEL RIESGO PERINATAL: INSTRUMENTOS
}

\author{
Instruments for identifying perinatal risk and \\ associated neuropsychological evaluation. \\ Santiago López-Gómez, Ph.D*, Carlos José Cajal-Cernuda, M.D.**, \\ Sonia $M^{a}$ Ordónez-Blanco, BSc***, Ana Fernanda Uribe-Rodríguez, Ph.D**** \\ Recibido: julio 9/08 - Aceptado: octubre 14/08
}

\section{RESUMEN}

Algunas complicaciones maternas perinatales específicas pueden ser determinantes en el desarrollo infantil. Se constata que muchos niños con una amplia variedad de alteraciones han sufrido diversas complicaciones durante su desarrollo gestacional. Por lo tanto, los riesgos maternos ocurridos a lo largo del período pre-perinatal llevan asociados, en ocasiones, diferentes daños, sean neuropsicológicos, físicos y/o psicosociales. En el presente artículo se identifican los principales factores asociados al riesgo perinatal y sus implicaciones, haciendo un recorrido por los procedimientos estandarizados de evaluación del riesgo, desde la perspectiva médiconeuropsicológica y psicosocial del neonato.

Palabras clave: riesgo perinatal, neuropsicología, procedimientos de evaluación, dimensiones de riesgo perinatal, desarrollo infantil.

\section{SUMMARY}

Some specific perinatal maternal complications can become key factors in child development. It is known that many children having a wide variety

* Doctor en Psicología. Facultad de Psicología, Universidad de Santiago de Compostela. Coruña. España.

** Médico de familia. Servicio Gallego de Salud (SERGAS). Centro de Salud Matogrande, Coruña, España.

*** Maestra de Educación Infantil, Xunta de Galicia. Coruña. España.

****Doctora en Psicología, Departamento de Ciencias Sociales, Pontificia Universidad Javeriana. Cali, Colombia. Correo electrónico: auribe@edu. puj.co of alterations have suffered different complications during their gestational development. The maternal risks which have taken place throughout the preperinatal period thus sometimes entail associated different damage which can be neuropsychological, physical and/or psycho-social. This report identifies the main factors associated with perinatal risk and their implications; the standard procedures for risk evaluation are shown from a medical-neuropsychological perspective and the newborn's psycho-social viewpoint.

Key words: perinatal risk, neuropsychology, evaluation procedure, perinatal risk dimension, child development.

\section{INTRODUCCIÓN}

A lo largo del ciclo del desarrollo humano, ningún período está marcado por cambios tan sustanciales como los nueve meses que median entre la concepción y el nacimiento. ${ }^{1}$ En este corto espacio temporal se reúnen un conjunto de factores que pueden dañar el desarrollo y producir problemas de salud en el feto y en su madre. Algunos problemas específicos y ciertas complicaciones médico-obstétricas son determinantes para el desarrollo en la primera infancia. Al respecto, también se constata la existencia de toda una serie de problemas que en ocasiones suelen pasar desapercibidos, al ser menos obvios, y manifestarse más tardíamente a modo de importantes 
afectaciones cognitivas, intelectuales o en el estado general del salud del niño.

Distintos estudios clínicos, tanto retrospectivos ${ }^{2-5}$ como prospectivos, ${ }^{6-9}$ muestran que muchos niños con una amplia variedad de problemas en el desarrollo han sufrido diversos riesgos y complicaciones perinatales. Desde este planteamiento, los niños que han vivido complicaciones durante el período prenatal, durante el parto, o bien en los primeros días del nacimiento, muestran un riesgo considerable frente a trastornos físicos, neuropsicológicos, cognitivos y comportamentales, que se ponen de manifiesto en toda una amplia gama de alteraciones médicas y psicológicas en su desarrollo. ${ }^{10,11}$

Estas complicaciones se pueden traducir, desde el punto de vista psicológico, como alteraciones cognitivas y comportamentales, ${ }^{12}$ de manera genérica, y muy en particular, en problemas específicos del desarrollo y en la adquisición de ciertas habilidades (p. ej. cálculo, lectura, motricidad). ${ }^{6}$ Por ello, el efecto de ciertos eventos ocurridos a muy temprana edad puede marcar de manera importante el desarrollo posterior, además de relacionarse con trastornos y síndromes complejos como autismo, esquizofrenia, déficits neurológicos, parálisis cerebral, etc. que, con toda seguridad, van a mermar la calidad de vida, tanto de estas personas, como la de todos los que le rodean.

A lo largo del presente artículo teórico ${ }^{13}$ se revisan algunas cuestiones relativas al riesgo perinatal, que se inician describiendo e identificando el riesgo perinatal y sus implicaciones, continuando con un recorrido por algunos instrumentos estandarizados de evaluación del riesgo, tanto desde la aproximación médico-neurológica, como desde la psicosocial.

\section{El riesgo perinatal}

Las investigaciones referidas al embarazo y a los riesgos y complicaciones perinatales han recibido una atención notable en las últimas décadas. Pese a ello, son todavía muchos los niños que experimentan complicaciones y experiencias de riesgo, tales como hipoxia, prematuridad, bajo peso, entre otras, y que suponen una posibilidad de alterar su desarrollo y su propia supervivencia. Al respecto, un alto número de niños que se encuentran en el grupo de los neonatos de riesgo, han llegado a sufrir trastornos en su desarrollo, consecuencia directa de esas complicaciones perinatales. ${ }^{14}$ En esta línea se informa que cerca del 65\% de estos niños, valorados como de riesgo, son derivados a servicios de educación especial antes de cumplir 10 años. ${ }^{15}$ Otros estudios indican que en los niños que han manifestado problemas en el desarrollo, se han encontrado cifras más altas de complicaciones perinatales que en otros grupos. ${ }^{5,16}$ Por ello, es necesario considerar el posible efecto adverso que, a nivel neuropsicológico, supone el impacto de las complicaciones perinatales en neonatos que experimentan determinados eventos durante el desarrollo gestacional.

En un sentido amplio, las complicaciones perinatales podrían definirse como problemas del embarazo, parto y período postparto, así como otras variables, sobre todo maternas, que son anteriores al embarazo, tales como la edad, el historial obstétrico y su estado general de salud. De esta manera, se habla de los factores de riesgo perinatales, refiriéndose a ellos como acontecimientos comprometidos que ocurren a lo largo del período perinatal, que se extiende desde la semana 12 de gestación hasta el día 28 de vida del neonato. ${ }^{10}$ Estos riesgos pueden derivar en déficits en el desarrollo y en alteraciones comportamentales. Generan, en muchos casos, complicaciones perinatales definidas como “... un término amplio para describir una gran variedad de circunstancias médicas comprometedoras que ocurren alrededor del tiempo del nacimiento, incluyendo el período prenatal y postnatal, al igual que los acontecimientos que se producen en el parto y el alumbramiento". ${ }^{17}$

Son muchas las investigaciones que apuntan, de manera directa, a la posibilidad de una relación entre determinados acontecimientos que tienen lugar durante el embarazo y las alteraciones en el posterior desarrollo infantil. ${ }^{12,18-21}$ No obstante, la literatura no es concluyente con el papel que juegan estas complicaciones perinatales en el origen de determi- 
nados trastornos durante el desarrollo. El resultado neuropsicológico de los niños expuestos a ellas es a menudo poco claro, pues en él convergen multitud de variables que son difíciles de controlar y precisar con exactitud, si bien se constata su afectación en el propio desarrollo fetal y neonatal. ${ }^{22}$

Los resultados encontrados hasta el momento son consistentes con la hipótesis de que algunos factores de riesgo son responsables de un amplio grupo de alteraciones comportamentales ${ }^{23-25}$ y emocionales, ${ }^{12}$ así como déficits cognitivos, ${ }^{26}$ dificultades educativas ${ }^{17}$ y de aprendizaje, ${ }^{27-29}$ trastornos psiquiátricos, ${ }^{11,24,30,31}$ alteraciones neuropsicológicas, ${ }^{32-34}$ daños y lesiones físicas diversas..$^{35,36}$ Un amplio grupo de investigaciones apuntan, de manera directa, a la posibilidad de una relación entre determinados acontecimientos que tienen lugar durante el embarazo y las alteraciones en el posterior desarrollo infantil. ${ }^{12,18-21}$ Diversos acontecimientos sucedidos durante la gestación se relacionan también con un anormal desarrollo cerebral y del SNC. ${ }^{37}$ Así pues, las investigaciones manifiestan que los acontecimientos y condiciones que ocurren durante el período perinatal tienen fuertes implicaciones en el posterior desarrollo físico, neurológico y psicosocial. ${ }^{21,38-40}$ Por otro lado, algunos estudios concluyen que el proceso de parto, debido a las hemorragias intracraneales, al uso de fórceps, a la anestesia, al sufrimiento fetal, y sobre todo a la asfixia perinatal, cuyas manifestaciones neurológicas constituyen la causa de la afectación cerebral y de secuelas neurológicas en recién nacidos, son un importante factor de riesgo que se puede asociar con múltiples daños. ${ }^{41,42}$ Ahora bien, es necesario contar con estudios que controlen el efecto de todas estas condiciones, de forma aislada y asociada, y poder así ser concluyentes.

Por esta razón, el impacto potencial que suponen los riesgos perinatales deberá ser considerado y evaluado, si fuese posible con anterioridad al embarazo, al igual que durante y después del mismo. ${ }^{12,24,43}$

Distintos estudios han estimado que la prevalencia de los embarazos que presentan riesgos perinatales se sitúan alrededor del 8 y 10\% e incluso con tasas más elevadas, cercanas al 15\%. ${ }^{44,45}$ La alta incidencia de problemas en el desarrollo, sugiere que determinadas dimensiones o factores específicos de estas complicaciones perinatales pueden estar influenciando el mismo, haciéndose patente, en ocasiones, de forma tardía en la adolescencia y en la edad adulta. ${ }^{31} \mathrm{Al}-$ gunos autores subrayan que la variedad de déficits neuropsicológicos, físicos y cognitivos continúan mostrándose como secuelas de las complicaciones pre-peri- y neonatales. ${ }^{46}$ Así pues, la severidad del daño causado dentro de una amplia variabilidad, va a estar influenciado desde la diversidad y multiplicidad de los riesgos afrontados.

El crecimiento físico, intelectual y, paralelamente, la calidad de vida del niño, van a depender de su normal desarrollo. A menudo, la interrupción de la secuencia evolutiva, debido a traumatismos, infecciones, alteraciones genéticas u otras causas, podría comprometer el crecimiento. ${ }^{11,14,24,47}$

El término factor de riesgo, desde este punto de vista perinatal, debería, por lo tanto, utilizarse de manera inequívoca, cuando se haya asociado con un problema posterior. Se debe entender desde una perspectiva biofisiológica y sanitaria, así como desde la consideración de las dimensiones psicosocial, económica y cultural.

\section{Evaluación del riesgo perinatal}

La valoración y el posterior seguimiento de los pacientes con riesgo perinatal tienen como objetivo fundamental minimizar, en la medida de lo posible, el efecto del mismo, tanto en la madre como en el futuro desarrollo de su hijo; además de identificar de forma temprana a los niños que previsiblemente pueden llegar a experimentar déficits evolutivos, a fin de proporcionar una atención apropiada antes de que se produzca un fracaso importante en el desarrollo, y poder optimizar de esta manera sus potencialidades, reduciendo el impacto del deterioro biofisiológico en favor de las intervenciones psicoeducativas tempranas. 
Bajo esta perspectiva, los cuidados prenatales deben considerar la inclusión, tanto de las atenciones físicas, como de los factores psicológicos, para contribuir a la prevención primaria de los trastornos mentales. ${ }^{48}$ Se trata, en definitiva, de incidir en todos los riesgos desde una perspectiva holística. ${ }^{47-51}$

Tradicionalmente, el posible retraso en el desarrollo ha sido valorado de dos maneras. ${ }^{52}$ La primera de ellas, utilizada sobre todo por personal médico y sanitario, justo después del parto, trata de constatar los factores de riesgo dentro del conocimiento existente sobre su asociación con posteriores déficits. Un segundo método de identificación, más cercano a la línea de los profesionales de la salud mental y la educación para la salud, valora el progreso madurativo del niño a partir de los comportamientos observados en él, en función de su edad cronológica y de su grupo normativo de referencia.

Tanto en la exploración médica como en la psicosocial, se deben poner en relación las variables que pueden conjugar el riesgo perigestacional con el ajuste neuropsicológico del neonato. Si bien se podrían efectuar mediciones a partir de criterios y observaciones no estandarizadas, absolutamente válidas y en ocasiones muy acertadas, se hace hincapié en este momento en las valoraciones que permiten determinadas escalas neonatales. Se indica, así mismo, que siempre se podrá profundizar en los juicios utilizando otros instrumentos no normativos o complementar varias mediciones, buscando una valoración más ajustada a una realidad concreta.

\subsection{Evaluación médica y neurropsicológica}

La evaluación médico-neuropsicológica permite medir y diagnosticar, desde un punto de vista médico, el funcionamiento del neonato. El examen neurológico neonatal explora la integridad y madurez del SNC. ${ }^{43}$ La idea predominante es que esta medida es un reflejo del estado de las estructuras corticales. Hay evidencias que ponen de manifiesto que mediante estas exploraciones las funciones corticales son evaluadas de manera concomitante, como podría ser a partir de la actividad motora espontánea u otras conductas similares. ${ }^{34}$ Esta evaluación también valora los comportamientos, tanto automáticos como voluntarios del recién nacido, y determina su habilidad para hacer frente a estímulos externos.

A continuación se describen una serie de escalas dentro de las más usadas y reconocidas a nivel internacional. Se incluyen, por otro lado, algunas de las escalas y guías de evaluación más utilizadas, tanto en la práctica clínica como en la investigación. La selección de las mismas se ha realizado de acuerdo a su amplitud de uso, aplicabilidad, reconocimiento por la comunidad científica y presencia en publicaciones de alto impacto. Los instrumentos deben aplicarse siempre bajo las condiciones que describe su protocolo. Para ello, se utilizará aquella/s que más se ajuste a los objetivos concretos de trabajo y en función de la experiencia clínica y diagnóstica en su manejo. Cabe señalar que muchos de ellos no están validados en nuestro medio, aún así, podrían utilizarse como complemento de otras pruebas, con el objetivo de afinar los datos obtenidos.

\section{Apgar Scoring System ${ }^{53,54}$}

El test de Apgar ha sido diseñado, en su formato original, para valorar de forma rápida el estado de ciertas dimensiones del recién nacido, justo en el momento de su nacimiento. En la actualidad, su aplicación se ha extendido a muchos países debido, sobre todo, a su simplicidad práctica y a su utilidad predictiva, a mediano y largo plazo, de posibles daños en el niño, especialmente neurológicos. ${ }^{55}$ Para su puntaje, el recién nacido se somete a cinco valoraciones (actividad -tono muscular-, pulso -frecuencia cardíaca-, movilidad -reflejos-, apariencia -color de la piel-, y respiración -movimientos respiratorios-), cada una de ellas puntuadas como "0", “1”, ó “2”, en dos momentos distintos, al minuto y a los cinco minutos de vida. La puntuación máxima será de 10, considerándose como normal la obtención de 7 puntos a los 5 minutos. ${ }^{51}$ Aunque en la actualidad la aplicación del Apgar sea una rutina médica, deberá 
siempre ser realizada por personal calificado, para recoger todo su potencial.

Algunos autores han estudiado la posibilidad de utilizar el Apgar como predictor de la mortalidad y la morbilidad postnatal. ${ }^{56}$ Encuentran al respecto, que las puntuaciones efectuadas en el primer minuto se muestran como buenas predictoras de la mortalidad neonatal, mientras que las puntuaciones realizadas a los cinco minutos van a predecir posibles daños neurológicos. ${ }^{56}$

Sería conveniente que la prueba se realizase de modo conjunto a otras pruebas clínicas (Rh, grupo sanguíneo, bilirrubina, etc.), y nunca como criterio diagnóstico único. ${ }^{57}$ La evaluación del Apgar también puede llevarse más allá de los cinco minutos, muy poco extendida en nuestro país, para identificar a bebés que presentan ciertos problemas, como puede ser la asfixia neonatal, asociándose con un mal pronóstico si persiste una puntuación baja más allá de los diez minutos. ${ }^{56,58}$

\section{Neonatal Behavior Assessment Scale (NBAS) ${ }^{59,60}$}

La Neonatal Behavior Assessment Scale (NBAS), también conocida como "Escala Brazelton", es una de las más utilizadas para valorar al neonato, desde el punto de vista neurológico y comportamental. La prueba consta de dos partes fundamentales. La primera valora, a partir de sus 37 ítems, algunos parámetros conductuales. La segunda parte, en sus 9 ítems, se centra en la exploración neurológica del bebé. Cada uno de los ítems se puntúa en una escala de 9 puntos. La prueba se agrupa en "clusters" de ítems que representan rasgos o características del comportamiento neonatal, y son: habituación, orientación, motricidad, variabilidad de estados, regulación del estado, estabilidad del sistema nervioso vegetativo y reflejos. La evaluación del bebé deberá realizarse sobre el tercer día de vida, en una habitación tranquila, con temperatura adecuada, luz tenue y entre dos tomas alimenticias.

El último objetivo es la evaluación del comportamiento interactivo y general del bebé, en términos de competencias y capacidades, buscando siempre optimizar la interacción del niño con el examinador. ${ }^{61}$ La escala se muestra sensible para detectar alteraciones de conducta en el neonato. Puede ser buena predictora a la hora de valorar comportamientos de riesgo perinatal, debido a que valora el efecto provocado en el neonato de distintos factores de riesgo: materno, uso de anestesia, desarrollo, sufrimiento fetal, etc. ${ }^{61}$

\section{Prechtl Neurological Examination of the Full-Term Newborn Infant ${ }^{62}$}

Trata de detectar las condiciones del sistema nervioso y la existencia de posibles alteraciones neurológicas en el neonato, recién nacido a término o pretérmino. Consta de 42 ítems que se agrupan en: estado, postura, movimientos espontáneos, temblor, ojos, reflejos y respuestas. Cada uno de los ítems se valora en función de un criterio dicotómico de estado óptimo / no óptimo.

\section{Transition Scale 63 $^{63}$}

La Transition Scale no es un cuestionario de amplia difusión, pese a ello, se incluye aquí por su consistencia y principios teóricos en los que se fundamenta. Parte de la estructura del Apgar, sin embargo, está específicamente diseñada para ofrecer información clínica completa relativa a un paciente, al considerar las mediciones realizadas después de 30 minutos del nacimiento. ${ }^{52}$ Utiliza un formato de presencia / no presencia para evaluar el estado del neonato y minimizar posibles complicaciones en las valoraciones intermedias, así como otras influencias clínicas externas. Los ítems que componen la escala están extraídos de las rutinas realizadas por el personal sanitario en el parto, como medidas de peso, temperatura, color, etc. Incluye un amplio número de condiciones que habitualmente se asocian con causas de mortalidad y morbilidad neonatal, como bajo peso, asfixia, presencia de meconio en el líquido amniótico, ruptura prematura de membranas, necesidad de oxígeno y otras. La escala, según sus autores, tiene un gran potencial para predecir la mortalidad infantil y la morbilidad neurológica asociada al riesgo gestacional. ${ }^{52,63}$ 


\section{Maternal Perinatal Scale (MPS) ${ }^{64,65}$}

La Maternal Perinatal Scale está diseñada para predecir la presencia de riesgos perinatales que se podrían asociar con déficits y alteraciones posteriores en el desarrollo del bebé. Se compone de 47 ítems, a modo de autoinforme, que las madres deben completar en función de las diversas preguntas que se les plantean. Algunas investigaciones ${ }^{65}$ manifiestan que esta información resulta ser muy consistente con los historiales clínicos pertenecientes a estas madres. La prueba hace un recorrido por un amplio número de factores biológicos y del desarrollo, que se pueden relacionar con posteriores alteraciones neurológicas; con lo cual, ante la presencia de los mismos, se podrían predecir ciertas dificultades, que se llegan a estimar en un $82 \%$ de éxito. ${ }^{10}$ Mantiene una tasa de estabilidad test-retest que supera el $0,90 .^{10}$

\section{Neonatal Neurological Examination (NEONEURO) ${ }^{66}$}

Ofrece una medida del estado neurológico y comportamental de las ejecuciones del neonato y recién nacido a término. Se compone de 32 ítems que se agrupan en torno a 7 factores: hipertonía, reflejos primarios, tono límbico, reflejos y temblores, estado de alerta y nerviosismo.

\section{Neonatal Neurobehavioral Examination (NNE) ${ }^{67}$}

Describe los cambios en el funcionamiento neurológico y comportamental, producto de la maduración del neonato o del recién nacido de alto riesgo. Consta de 27 ítems que describen el funcionamiento a nivel motor, del tono muscular y de los reflejos primarios.

\section{Assessment of Preterm Infant's Behavior (APIG) ${ }^{68}$} Se fundamenta en la escala NBAS de Brazelton. Mide el comportamiento, su organización, funcionamiento e integración de neonatos pretérmino en diversos sistemas: fisiológico, motor, estado, atención-interacción y regulación.

\section{Test of Infant Motor Performance (TIMP) ${ }^{69}$}

Valora una serie de componentes del control del movimiento del neonato. Tiene dos partes: la pri- mera, compuesta por 27 observaciones del comportamiento espontáneo, y la segunda, con 25 ítems de comportamientos elicitados. Los factores que se describen son: orientación de la cabeza en el espacio, respuesta a estímulos visuales y auditivos, alineación y manejo del cuerpo, y control del movimiento de brazos y piernas.

\section{Neurobehavioral Assessment of the Preterm Infant $(\text { NAPI })^{70}$}

Mide algunas dimensiones en bebés nacidos antes de término, de su desarrollo y progresos de las funciones neurocomportamentales. Las dimensiones que incluye son: vigor y desarrollo motor, control del cuello y ángulo, orientación y alerta, irritabilidad, llanto, sueño y estado del comportamiento.

\section{Neurological Assessment of the Preterm and Full- term Newborn Infant (NAPFI) ${ }^{71}$}

Se compone de 33 ítems que abarcan cuatro secciones: habituación, movimiento y tono, reflejos y respuestas neurocomportamentales. Realiza un examen secuencial del neonato, utilizando un sistema de medida de fácil manejo y con cuantificaciones objetivas.

\subsection{Evaluación psicológica y social}

Sin duda, la evaluación psicológica ha de relacionarse estrechamente con la neurológica, por el hecho de constatarse que los déficits comportamentales y del desarrollo, interaccionan con las disfunciones cerebrales a modo de retroalimentación. La estimación del nivel de ajuste psicológico del neonato, expuesto a un riesgo obstétrico, va a fundamentar la necesidad de realizar una completa exploración.

Existen diversas pruebas y escalas que permiten efectuar un barrido por las conductas neonatales, e incluso valorar dimensiones de naturaleza social que se relacionan con el desarrollo perinatal.

Los test y guías descritos a continuación, permiten valorar el estado general del comportamiento y ciertas condiciones sociales en el neonato desde los primeros días del nacimiento, y tienen un carácter predictivo y de seguimiento en su desarrollo. Sólo 
se hace referencia a algunas de las más utilizadas, considerando su importancia, facilidad de manejo, información aportada y uso prolongado.

\section{Escalas Gesell ${ }^{72}$}

Arnold Gesell ha construido una serie de escalas para valorar el desarrollo del lactante y del niño de máximo 5 años. Parten de la observación de ciertas capacidades y comportamientos, tratando de predecir el futuro de su desarrollo. Incluyen observaciones genéricas de conductas físicas (motrices), sociales e intelectuales.

\section{Escalas Bayley de desarrollo infantil (BSID) $^{73}$}

La prueba, a través de sus tres escalas, permite evaluar el desarrollo temprano del niño hasta los dos años y medio. Valora aspectos relacionados con el desarrollo cognitivo y comunicativo (escala mental), el grado de coordinación corporal y de las actividades psicomotrices (escala de psicomotricidad), y las orientaciones sociales y hacia el entorno (registro del comportamiento). Permite, a su vez, hacer una valoración del posible retraso a nivel lingüístico e intelectual.

\section{Denver Developmental Screening Test (DDST) ${ }^{74}$}

El "DDST" valora un conjunto de capacidades en el bebé, desde las 2 semanas de vida hasta los 6 años. Incluye medidas de motricidad fina, motricidad gruesa, lenguaje, respuestas sociales y personales. La información predictiva sobre cualquier alteración del desarrollo es escasa, aunque permite una buena aproximación a nivel de la inteligencia y del desarrollo del niño.

\section{BATELE, inventario de desarrollo ${ }^{75}$}

Evalúa algunas de las actividades fundamentales del niño, desde su nacimiento hasta los 8 años, en diversas áreas del desarrollo: personalidad/social, adaptativa, motora, comunicación y cognitiva, permitiendo valorar su progreso y diagnosticar posibles deficiencias o retrasos. Consta de más de
300 elementos, que se pueden aplicar en su versión completa o abreviada, obteniendo información de tres tipos: mediante un examen estructurado; mediante una guía de observación, tanto en clase como en casa, y mediante información aportada por los padres y profesores.

\section{Currículo Carolina. Evaluación y ejercicios para bebés y niños pequeños con necesidades especiales $^{76}$}

Se trata de un programa que consta de 26 secuencias lógicas que abarcan 5 áreas principales: cognición, comunicación, adaptación social, motricidad fina y motricidad gruesa. Su aplicación permite crear programas para las necesidades específicas de cada niño, incluso con limitaciones leves, moderadas o múltiples; adaptando los ejercicios a los niños con estas limitaciones e integrándolas en actividades y rutinas de la vida cotidiana. Posibilita un seguimiento del niño, desde su nacimiento hasta el nivel de desarrollo correspondiente con 24 meses, valorando los posibles retrasos y su significatividad, sobre todo desde el punto de vista de la adaptación del currículo y también a nivel terapéutico.

\section{Portage, guía de educación preescolar $^{77}$}

Se trata de una guía integrada por 578 fichas que corresponden a 6 áreas: general, socialización, lenguaje, autoayuda, cognición y desarrollo motriz. Ofrece una descripción del desarrollo de diversas áreas de estas aptitudes infantiles, desde el nacimiento hasta los 6 años, con el fin de programar actividades para conseguir mayores objetivos.

\section{Escala observacional del desarrollo $(\text { EOD })^{78}$}

La escala permite una descripción del desarrollo evolutivo a partir de la observación de diversas áreas, como: afectividad, desarrollo físico, desarrollo sensorial, coordinación motriz y perceptivo-motriz, 
comunicación, inteligencia y otras. Se compone de cuestionarios de aplicación y fichas de perfil para las distintas edades, desde los "0" a los "17" años, así como fichas breves que permiten a los padres la calificación de sus hijos. Además de la función evaluativa y explicativa de los procesos evolutivos, se proponen medidas de intervención correctoras o estimuladoras para activar los comportamientos observados.

\section{CONCLUSIONES}

A través de la revisión de diversas investigaciones, se ha puesto de manifiesto que muchos problemas neuropsicológicos y comportamentales muestran correlaciones significativas con la presencia de riesgos y complicaciones perinatales.

Existe al respecto una alta incidencia de niños con problemas en el desarrollo, en los cuales se han hallado, a través de análisis retrospectivos de sus historiales médicos, cifras más altas de complicaciones perinatales que en otros grupos poblacionales. Las variables incidentes son múltiples y difíciles de controlar, pero suponen un factor de riesgo importante frente a múltiples alteraciones, no sólo físicas, sino también neuropsicológicas y psicosociales.

Muchos factores de riesgo perinatales influencian de modo directo el embarazo, de tal manera que incrementan el riesgo frente a diversas patologías que se ponen de manifiesto con el desarrollo.

La evaluación del riesgo perinatal y de su resultado, permite evidenciar de forma temprana la relación entre un fenómeno perinatal y el resultado final. En la actualidad existen múltiples instrumentos que evalúan, tanto el estado de las estructuras corticales, como los comportamientos automáticos y voluntarios del recién nacido, como el ajuste psicosocial, y que permiten conocer no sólo cuál es el resultado neonatal, sino también poder minimizar los posibles riesgos sufridos en el embarazo.

\section{REFERENCIAS}

1. Vasta R, Haith MM, Miller SA. Psicología infantil. Barcelona: Ariel; 1996. p. 3-11.

2. Cohen SE, Parmelee AH, Sigman M, Beckwith L. Neonatal risk factors in preterm infants. Appl Res Ment Retard 1982;3:265-78.

3. Lobel M. Conceptualizations, meaurement, and effects of prenatal maternal stress on birth outcomes. J Behav Med 1994;17:225-72.

4. Meier A. Child psychiatric sequelae of maternal war stress. Acta Psychiatr Scand 1985;72:505-11.

5. Wilkerson DS, Volpe AG, Dean RS, Titus JB. Perinatal complications as predictors of infantile autism. Int J Neurosci 2002;11:1085-98.

6. González de Dios J, Moya M. Asfixia perinatal, encefalopatía hipóxico-isquémica y secuelas neurológicas en recién nacidos a término. II. Descripción e interrelaciones. Rev Neurol 1996;24:969-76.

7. Huizink AC, Robles PG, Mulder EJ, Visser GH, Buitelaar JK. Psychological measures of prenatal stress as predictors of infant temperament. J Am Acad Child Adolesc Psychiatry 2002;41:1078-85.

8. Lester BM, Tronick EZ, LaGasse L, Seifer R, Bauer CR, Shankaran S, et al. The maternal lifestyle study: effects of substance exposure during pregnancy on neurodevelopmental outcome in 1-month-old infants. Pediatrics 2002;110:1182-92.

9. Ramsay DS, Lewis M. The effects of birth condition on infants' cortisol response to stress. Pediatrics 1995;95:546-9.

10. Gray JW, Dean RS, Rattan G. Assessment of perinatal risk factors. Psychol Sch 1987;24:15-21.

11. Simonds JF, Aston L. Relationship between minor physical anomalies, perinatal complications, and psychiatric diagnoses in children. Psychiatry Res 1981;4:181-8.

12. Batchelor ES, Dean RS, Gray JW, Wenck S. Classification rates relative risk factors for perinatal events predicting emotional/behavioral disorders in children. Pre-and-Peri-Natal Pshycology Journal 1989;5:327-41.

13. Montero I, Leon OG. Sistema de clasificación del método en los informes de investigación en Psicología. Int J Clin Health Psychol 2005;5:115-27.

14. Gray JW, Dean RS, Strom DA, Wheeler TE, Brockley M. Perinatal complications as predictors of developmental disabilities. Dev Neuropsychol 1989;5:105-13. 
15. Nickel RE, Bennet FC, Lamson FN. School performance of children with birthweights of 1,000 $\mathrm{g}$ or less. Am J Dis Child 1982;136:105-10.

16. Gray JW, Dean RS. Behavioral implications of perinatal complications: an overview. En: Gray JW, Dean RS (eds.). Neuropsychology of perinatal complications. New York: Springer Publishing Company; 1991. p. $1-21$.

17. Telzrow CF. Impact of perinatal complications on education. En: Gray JW, Dean RS (eds.). Neuropsychology of perinatal complications. New York: Springer Publishing Company; 1991. p. 16185.

18. Avery G. Effects of social, cultural and economic factors on brain development. En: Freeman JM (ed.). Prenatal and perinatal factors associated with brain disorders. Bethesda: National Institutes of Health; 1985. p. 157 192.

19. Dawson G, Ashman SB, Carver LJ. The role of early experience in shaping behavioral and brain development and its implications for social policy. Dev Psychopathol 2000;12:695-712.

20. Decouflé P, Boyle CA, Paulozzi LJ, Lary JM. Increased risk for developmental disabilities in children who have major birth defects: a population-based study. Pediatrics 2001;108:728-34.

21. Kopp CB, Krakow JB. The developmentalist and the study of biological risk: a view of the past with an eye toward the future. Child Dev 1983;54:1086-1108.

22. Faden VB, Hanna E, Graubard BI. The effect of positive and negative health behavior during gestation on pregnancy outcome. J Subst Abuse 1997;9:63-76.

23. van den Boom D, Gravenhorst JB. Prenatal and perinatal correlates of neonatal irritability. Infant Behav Dev 1995;18:117-21.

24. Eaton WW, Mortensen PB, Thomsen PH, Frydenberg M. Obstetric complications and risk for severe psychopathology in childhood. J Autis Dev Disor 2001;31:279-85.

25. Weissman MM, Warner V, Wickramaratne PJ, Kandel DB. Maternal smoking during pregnancy and psychopathology in offspring followed to adulthood. J Am Acad Child Ann Psychiat 1999;38:892-9.

26. O'Callaghan M, Williams GM, Andersen MJ, Bor W, Najman JM. Social and biological risk factors for mild and borderline impairment of language comprenhension in a cohort of five-year-old children. Dev Med Child Neurol 1995;37:1051-61.
27. Hill SK, Cawthorne V, Dean RS. Utility of the Maternal Perinatal Scale (MPS) in distinguishing normal form learning disabled children. Int J Neurosci 1998; 95:141-54.

28. Korkman M, Liikanen A, Fellman V. Neuropsychological consequences of very low weight and asphyxia at tem: follow-up until school age. J Clin Exp Neuropsychol 1996;18:220-33.

29. Stanton C, Chapman D, Scott K. Identification of early risk factors for learning disabilities. Journal of Early Intervention 2001;24:193-206.

30. Adams W, Kendell RE, Hare EH, Munk-Jørgensen P. Epidemiological evidence that maternal influenza contributes to the aetiology of schizophrenia. An analysis of Scottish, English, and Danish data. Br J Psychiatry 1993;163:522-34.

31. Geddes JR, Verdoux H, Takei N, Lawrie SM, Povet P, Eagles JM, et al. Schizophrenia and complications of pregnancy and labor: an individual patient data metaanalysis. Schizophr Bull 1999;25:413-23.

32. Frield PA, Watkinson B, Dillon RF, Dulberg CS. Neonatal neurological status in a low-risk population after prenatal exposure to cigarettes, marijuana, and alcohol. J Dev Behav Pediatr 1987;8:318-26.

33. Jones NA, Field T, Fox NA, Davalos M, Lundy B, Hart S. Newborns of mothers with depressive symptoms are physiologically less developed. Infant Behav Dev 1998;21:537-41.

34. Prechtl HF. Qualitative changes of spontaneous movements in fetus and preterm infant are a marker of neurological dysfunction. Early Hum Dev 1990;23:151-8.

35. Berger R, Garnier Y. Perinatal brain injury. J Perinat Med 2000;28:261-85.

36. Koniak-Griffin D, Turner-Pluta C. Health risks and psychosocial outcomes of early childbering: a review of the literature. J Perinat Neonatal Nurs 2001;15:1-17.

37. Mendola P, Selevan SG, Gutter S, Rice D. Environmental factors associated with a spectrum of neurodevelopmental deficits. Ment Retard Dev D R 2002;8:188-97.

38. Field T, Diego MA, Dieter J, Hernández-Reif M, Schanberg S, Kuhn C, et al. Depressed vithdrawn and intrusive mother's effects on their fetuses and neonates. Infant Behav Dev 2001;24:27-39.

39. Maldonado-Durán JM, Helming L, Moody C. Signos de alarma en la detección temprana de problemas emocionales y conductuales en el bebé. Perinatología y Reproducción Humana 2001;15:21-30. 
40. Schothorst PF, Van Engeland H. Long-term behavioral sequelae of prematurity. J Am Acad Child Adolesc Psyquiatry 1996;35:175-83.

41. López Gómez L, Castro P, Bernardo AB, Megías A, Blanco D. Evaluación precoz en la hipoxia perinatal. Marcadores pronósticos. Rev Neurol 2003;31:1142-6.

42. Siegel LS. Reproductive, perinatal, and environmental variables as predictors of development of preterm (less than 1501 grams) and full term children at five years. Semin Perinatol 1982;6:274-9.

43. Majnemer A, Mazer B. Neurologic evaluation of the newborn infant: definition and psychometric properties. Dev Med Child Neurol 1998;40:708-15.

44. Behrman RE, Fanaroff AA, Martin RJ, Merkatz IR. Behrman's Neonatal-Perinatal medicine: diseases of the fetus and infant. St. Louis: CV Mosby Company; 1983.

45. Kurjak A, Vecek N, Hafner T, Bozek T, FundukKurjak B, Ujevic B. Prenatal diagnosis: what does four-dimensional ultrasound add? J Perinat Med 2001;30:57-62.

46. Gray JW, Dean RS, Lowrie RA. Relationship between socioeconomic status and perinatal complications. J Clin Child Psychol 1988;17:352-8.

47. Croen LA, Grether JK, Selvein S. Descriptive epidemiology of autism in a California population: who is at risk? J Autism Dev Disord 2002;32:217-24.

48. Misra DP, Guyer B, Allston A. Integrated perinatal health framework. A multiple determinants model with a life span approach. Am J Prev Med 2003;25:65-75.

49. Ward AJ. A comparison and analysis of the presence of family problems during pregnancy of mothers of autistics children and mothers of normal children. Child Psychiat Hum Dev 1990;20:279-88.

50. Bortman M. Factores de riesgo de bajo peso al nacer. Rev Panam Salud Publica 2002;3:314-21.

51. Sola A, Tapia JL. Evaluación del recién nacido. En: Pérez A, Donoso E (eds.). Obstetricia. Santiago de Chile: Mediterránea; 1999. p. 909-26.

52. Strom DA. Assessment and identification of perinatal risk factor. En: Gray JW, Dean RS (eds.). Neuropsychology of perinatal complications. New York: Springer Publishing Company; 1991. p. 109-27.

53. Apgar V. A proposal for a new method of evaluation of the newborn infant. Curr Res Anesth 1953;32:260-7.

54. Apgar V, James LS. Further observations on the newborn scoring system. Am J Dis Child 1962;104:419-29.
55. Goodwin TM. Role of the Apgar scores in assessing perinatal asphyxia. Contemp Pediatr 1997:142-52.

56. Nelson KB, Ellenberg JH. Apgar scores as predictors of chronic neurologic disability. Pediatrics 1981;68:36-44.

57. Quero J. Asfixia perinatal y reanimación del recién nacido. En: Usandizaga JA, Fuente $\mathrm{P}$ (eds.). Tratado de obstetricia y ginecología. Vol I: Obstetricia. Madrid: McGraw-Hill-Interamericana; 1977. p. 543-52.

58. Freeman J. Summary. En: Freeman J (ed.). Prenatal and perinatal factors associated with brain disorders. Bethesda, M.D.: National Institutes of Health; 1985. p. 437-50.

59. Brazelton TB. Neonatal Behavioral Assesment Scale. Clinics in Developmental Medicine, N ${ }^{\circ} 50$. Philadelphia: Lippincott; 1973.

60. Brazelton TB, Nugent K. Escala para la evaluación del comportamiento neonatal. Barcelona: Paidós; 1997.

61. Costas C, Botet F, Ortolá ME. Comportamiento del neonato de bajo peso nacido a término, según la escala Brazelton. An Esp Pediatr 1989;34:37-40.

62. Prechtl HF. The Neurological Examination of the Full-term Newborn Infant ( $2^{\mathrm{a}}$ ed.). Clinics in Developmental Medicine. No 63. London: Spastics International Medical Publications; 1977.

63. Strom DA. The Transition Scale: predicting neurological morbility at the time of bitrh. Dissertation Abstracts International. Muncie, Indiana: Ball State University; 1988.

64. Dean RS, Gray JW. Maternal Perinatal Scale. Muncie, Indiana: Ball State University; 1985.

65. Gray JW, Dean RS, Rattan G y Bechtel BA. Mother's self-report of perinatal complications. J Clin Child Psychol 1988;17:242-7.

66. Sheridan-Pereira M, Ellison PH, Helgeson V. The construction of a scored neonatal neurological examination for assessment of neurological integrity in full-term neonates. J Dev Behav Pediatr 1991;12:25-30.

67. Morgan AM, Koch V, Aldag J. Neonatal neurobehavioral examination: a new instrument for quantitative analysis of neonatal neurological status. Phys Ther 1988;68:1352-8.

68. Als H, Lester B, Tronick E, Brazelton TB. Manual for the Assessment of Preterm Infant's Behavior (APIB). En: Fitzgerald H, Lester B, Yogman M, (eds). Theory and Research in Behavioral Pediatrics. New York: Plenum Press; 1982. p. 65-132. 
69. Campbell S, Osten E, Kolobe T, Fisher AG. Development of the test of infant motor performance. Phys Med Rehabil Clin 1993;4:541-50.

70. Korner A, Thom VA. Neurobehavioral Assessment of the Preterm Infant. New York: The Psychological Corporation; 1991.

71. Dubowitz L, Dubowitz V. The neurological assessment of the preterm and full-term newborn infant. Clinics in Developmental Medicine. No 79. London: Spastics International Medical Publications; 1981.

72. Gesell A. Diagnóstico del desarrollo normal y anormal del niño: métodos clínicos y aplicaciones prácticas. Buenos Aires: Paidós; 1945.

73. Bayley N. Escalas Bayley de desarrollo infantil (BSID). Madrid: TEA; 1977.
74. Frankenburg WK, Dodds JB. The Denver developmental screening test. J Pediatr 1967;71:18191.

75. Newborg J, Stock JR, Wneck L. Batelle: Inventario de desarrollo. Madrid: TEA; 1989.

76. Johnson NM, Jens KG, Attermeier SM, Hacker BJ. Currículo Carolina. Evaluación y ejercicios para bebés y niños pequeños con necesidades especiales. Madrid: TEA; 1994.

77. Bluma SM, Shearer MS, Frohman AH, Hiliand IM. Portage, guía de educación preescolar. Madrid: TEA; 1995.

78. Secadas F. Procesos evolutivos y escala observacional del desarrollo: del nacimiento a la adolescencia. Madrid: TEA; 1992. 